\title{
“Coronary Confusion” Congenital Anomaly of the Left Main Coronary Artery Presenting as a Myocardial Infarction in a Young Female
}

\author{
Mohammed Al-Sadawi ${ }^{1}$, Hope A. Taitt, ${ }^{1}$ Michael Haddadin ${ }^{1}$, Shakil Shaikh ${ }^{1}$, \\ Murad Almasri ${ }^{2}$, Angeleque Hartt ${ }^{1}$, Samy I. McFarlane ${ }^{1, *}$ \\ ${ }^{1}$ Department of Internal Medicine, State University of New York: Downstate Medical Center, \\ Brooklyn, New York, United States-11203 \\ ${ }^{2}$ Department of Pediatrics, The University of Texas Medical Branch at Galveston, Galveston, Texas, United States-77555 \\ *Corresponding author: smcfarlane@downstate.edu
}

Received January 04, 2019; Revised March 01, 2019; Accepted March 20, 2019

\begin{abstract}
Coronary artery anomalies are rare congenital disorders occurring in 0.3-5.6 \% of the population. However, it carries a potential risk of sudden death in young athletes due to the development of premature coronary artery disease. The diagnosis of coronary artery anomalies is usually made incidentally; commonly found while investigating other cardiac conditions. A coronary CT angiography and traditional interventional angiography are the gold standards for diagnosing congenital coronary artery anomalies. Here, we present a case of a 27 year-old female with a history of hypertension and membranous glomerulonephritis with proteinuria presenting with an inferior ST elevation myocardial infarction. Cardiac catheterization revealed an anomalous origin of the left anterior descending artery from the right coronary cusp and absence of the left circumflex artery with a super-dominant right coronary artery. The patient had an occlusion of the right posterior descending artery and the right posterolateral segment. We discuss the clinical diagnosis and intervention of the case along with review of the literature.
\end{abstract}

Keywords: congenital coronary artery anomalies, myocardial Infarction in young, left main coronary artery anomaly

Cite This Article: Mohammed Al-Sadawi, Hope A. Taitt, Michael Haddadin, Shakil Shaikh, Murad Almasri, Angeleque Hartt, and Samy I. McFarlane, “'Coronary Confusion' Congenital Anomaly of the Left Main Coronary Artery Presenting as a Myocardial Infarction in a Young Female.” American Journal of Medical Case Reports, vol. 7, no. 3 (2019): 41-45. doi: 10.12691/ajmcr-7-3-3.

\section{Introduction}

Congenital coronary artery anomalies (CAAs) are relatively rare, with a prevalence of approximately $0.3 \%-5.6 \%$ [1]. It is associated with sudden death in young athletes [2]. Anomalous origins of the left anterior descending artery (LAD) from right coronary cusps and total absence of the left circumflex artery (LCX) with a super-dominant right coronary artery (RCA) is a rare congenital anomaly that may predispose to coronary ischemic events. We are presenting a 27 year-old female who came with an inferior ST elevation myocardial infarction with multiple coronary anomalies and suspicion of thrombophilia predisposing the aforementioned cardiac event.

\section{Case Presentation}

A 27 year-old African American female with a past medical history of pre-eclampsia during her first pregnancy, hypertension, bronchial asthma, and chronic kidney disease (CKD) due to membranous glomerulonephritis with proteinuria with no history of smoking or toxic habits, presented with a chief complaint of chest pain. She described it as severe, crushing and associated with diaphoresis. The pain did not improve with acetaminophen and asthma medications. The patient called EMS and was brought to the Emergency Department (ED). In the ED, physical examination revealed a young well developed female, anxious, in mild distress, due to chest pain. She was afebrile with a blood pressure of 129/81 and a heart rate of 69. Her electrocardiogram showed ST elevation in leads II, III, aVF (Figure 1). Her troponin I was elevated to $0.14 \mathrm{ng} / \mathrm{L}$ [Normal $<0.04 \mathrm{ng} / \mathrm{L}$ ]. Bedside echocardiography revealed an estimated ejection fraction of $45 \%$ and regional wall motion abnormalities in a multivessel territory. She was started immediately on aspirin and clopidogrel; STEMI code was initiated. She was immediately taken to cardiac catheterization which revealed the congenital absence of her left main coronary artery, an anomalous origin of the LAD from the right coronary cusp immediately superior to origin of the RCA. The RCA continued to supply the LCX territory with focal moderate ectasia. She had severe single vessel disease 
within her RCA territory: 100\% stenosis in the proximal third of right posterior descending artery (RPDA) and discrete $85 \%$ stenosis in right posterolateral segment (RPLS) just after the RPDA. She was treated with 2 drug eluting stents which deployed successfully in both lesions (Figure 2, Figure 3). Post cardiac catheterization transthoracic echocardiography revealed inferior and inferoseptal hypokinesis and ejection fraction estimated to be $40 \%$. CT coronary angiography showed that the RCA originates from the right coronary cusp, LAD coronary artery from right coronary cusp just adjacent to the origin of the RCA with a prepulmonic course. The RCA is super-dominant supplying the territory of the obtuse marginals and left circumflex with no vessel originating from the left or noncoronary cusps of the aortic root. (Figure 4, Figure 5) Blood tests revealed increase triglycerides $484 \mathrm{mg} / \mathrm{dl}$ [Normal range $<150 \mathrm{mg} / \mathrm{dl}$ ] and cholesterol $268 \mathrm{mg} / \mathrm{dl}$ [Normal Range $<200 \mathrm{mg} / \mathrm{dl}$ ] and decreased high-density lipoprotein (HDL) $25 \mathrm{mg} / \mathrm{dl}$ [Normal Range $>40 \mathrm{mg} / \mathrm{dl}$ ]. Thrombophilia profile was sent and revealed mild increase in homocysteine 14 $\mu \mathrm{mol} / \mathrm{L}$ [Normal Range 4-15 $\mu \mathrm{mol} / \mathrm{L}$ ] and positive Dilute Russell's Viper Venom Time. She was discharged in stable condition with appropriate follow up.

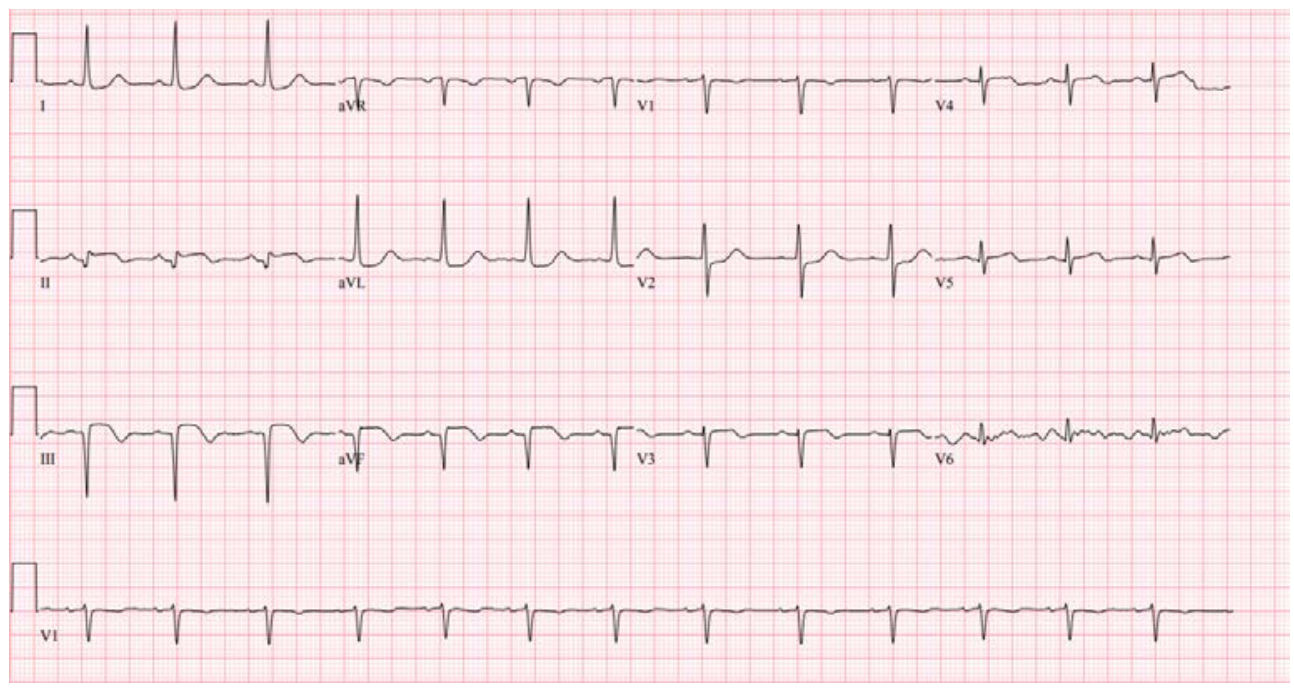

Figure 1. Electrocardiogram of the patient at presentation demonstrated ST segment elevation in leads II, III, aVF

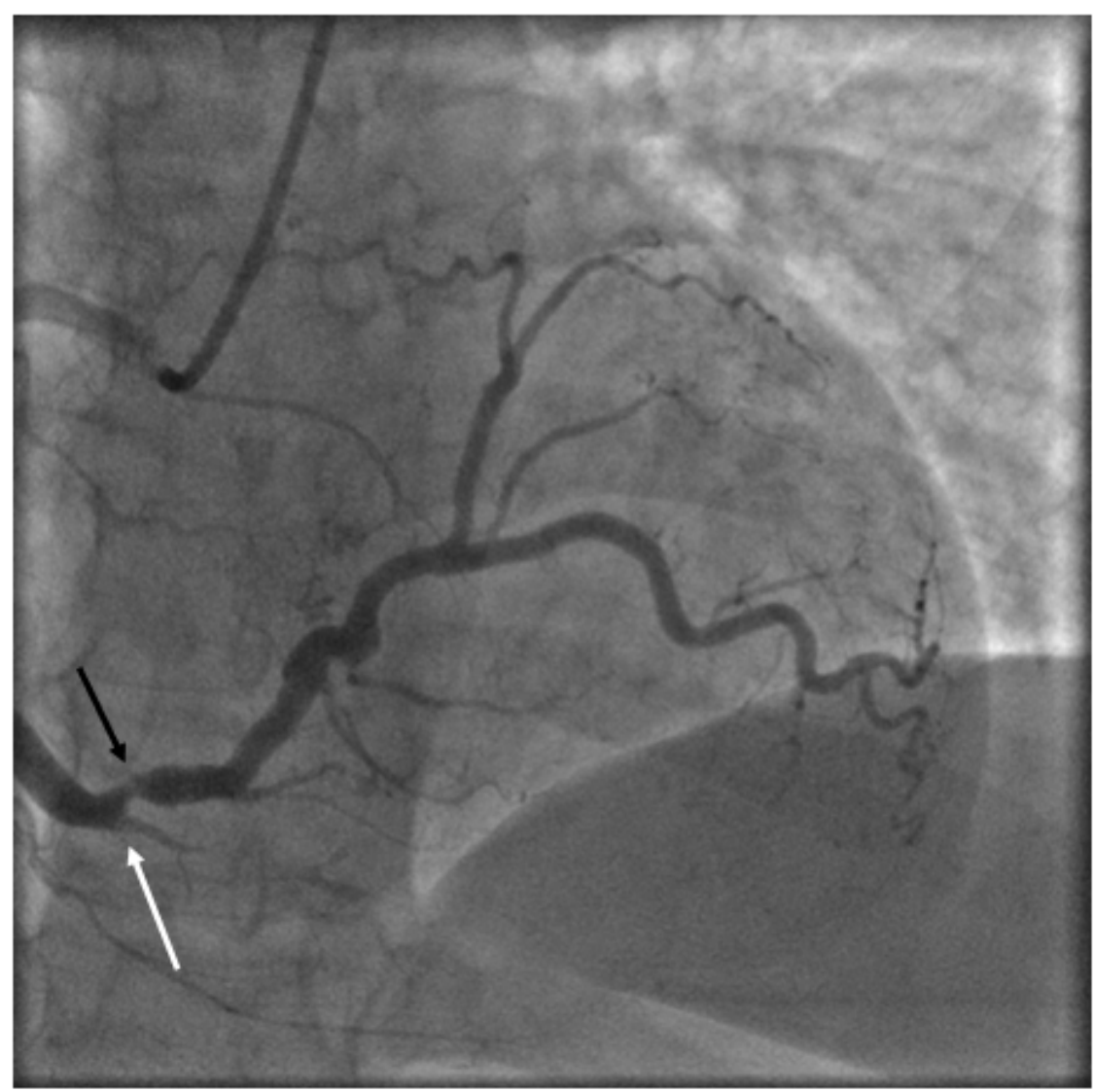

Figure 2. Cardiac catheterization revealed the right coronary artery (RCA) continues to supply the LCX territory with focal moderate ectasia. She has a severe single vessel disease at RCA territory: $100 \%$ stenosis in the proximal third of right posterior descending artery (RPDA) -black arrow- and discrete 85 \% stenosis in right posterolateral segment (RPLS) just after the RPDA - white arrow- 


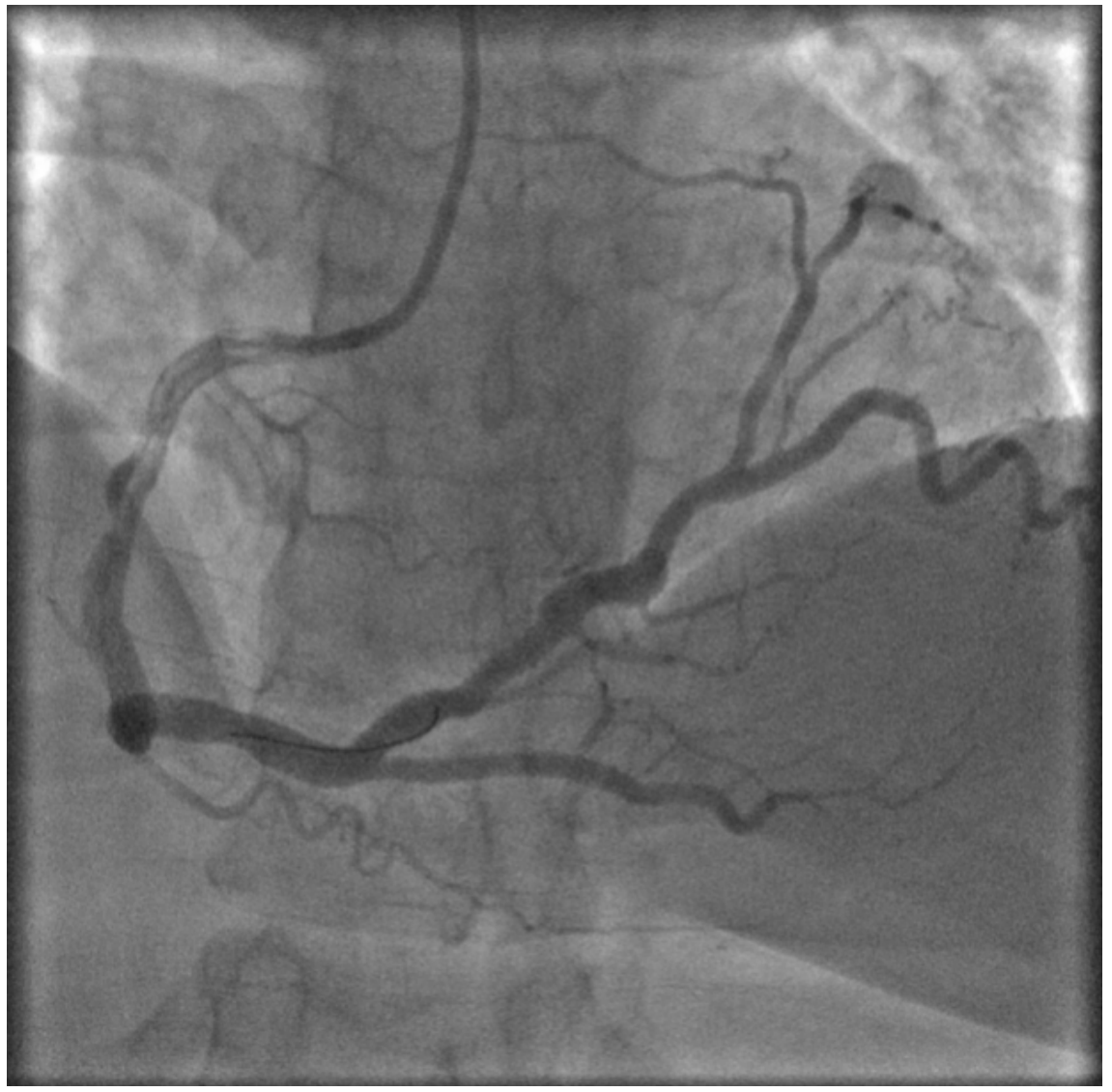

Figure 3. Cardiac catheterization showing the 2 drug eluting stents placed successfully in the right posterior descending artery (RPDA) and the right posterolateral segment RPLS

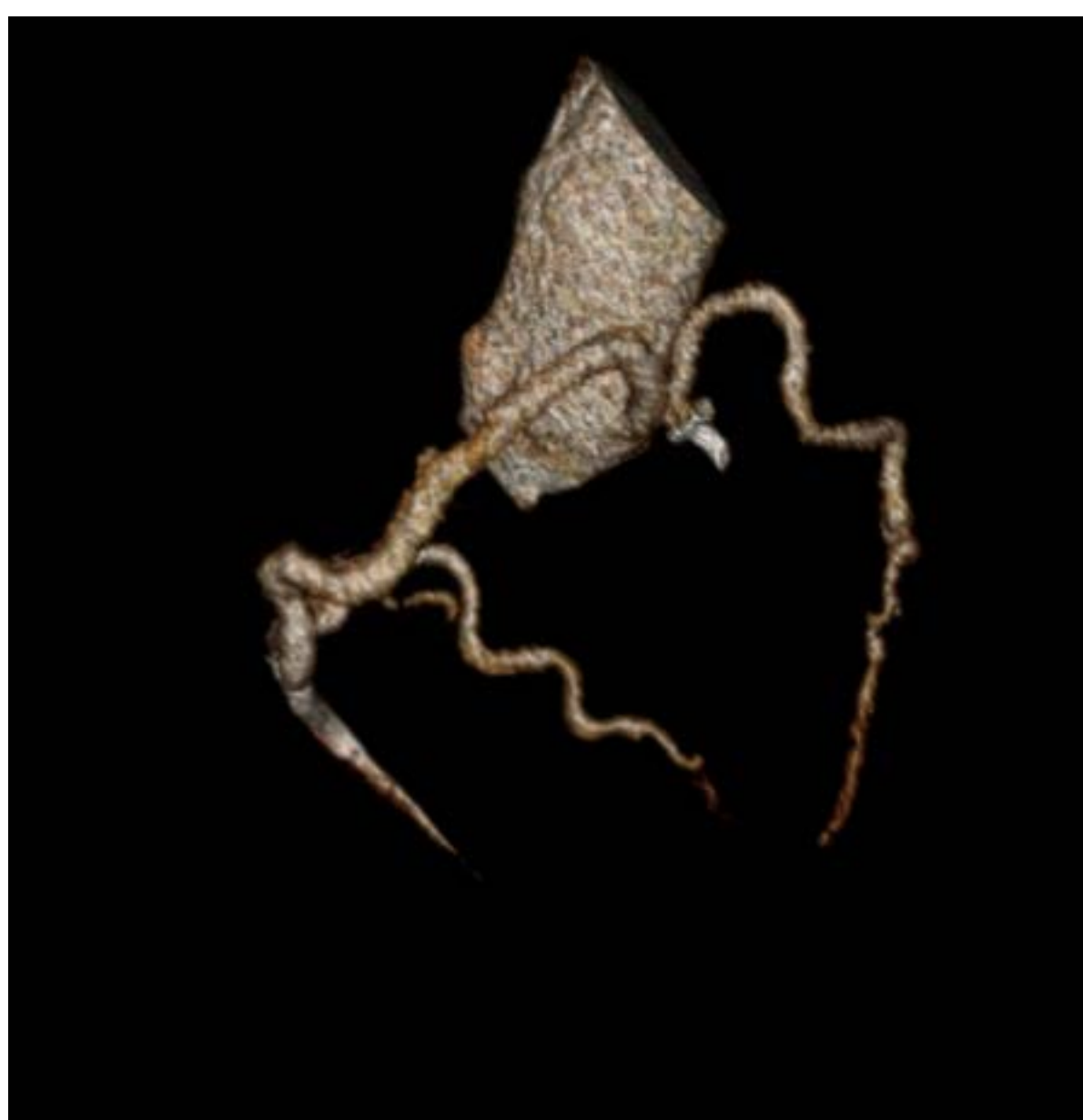

Figure 4. CT coronary angiography 3D Reconstruction of coronary vessels 


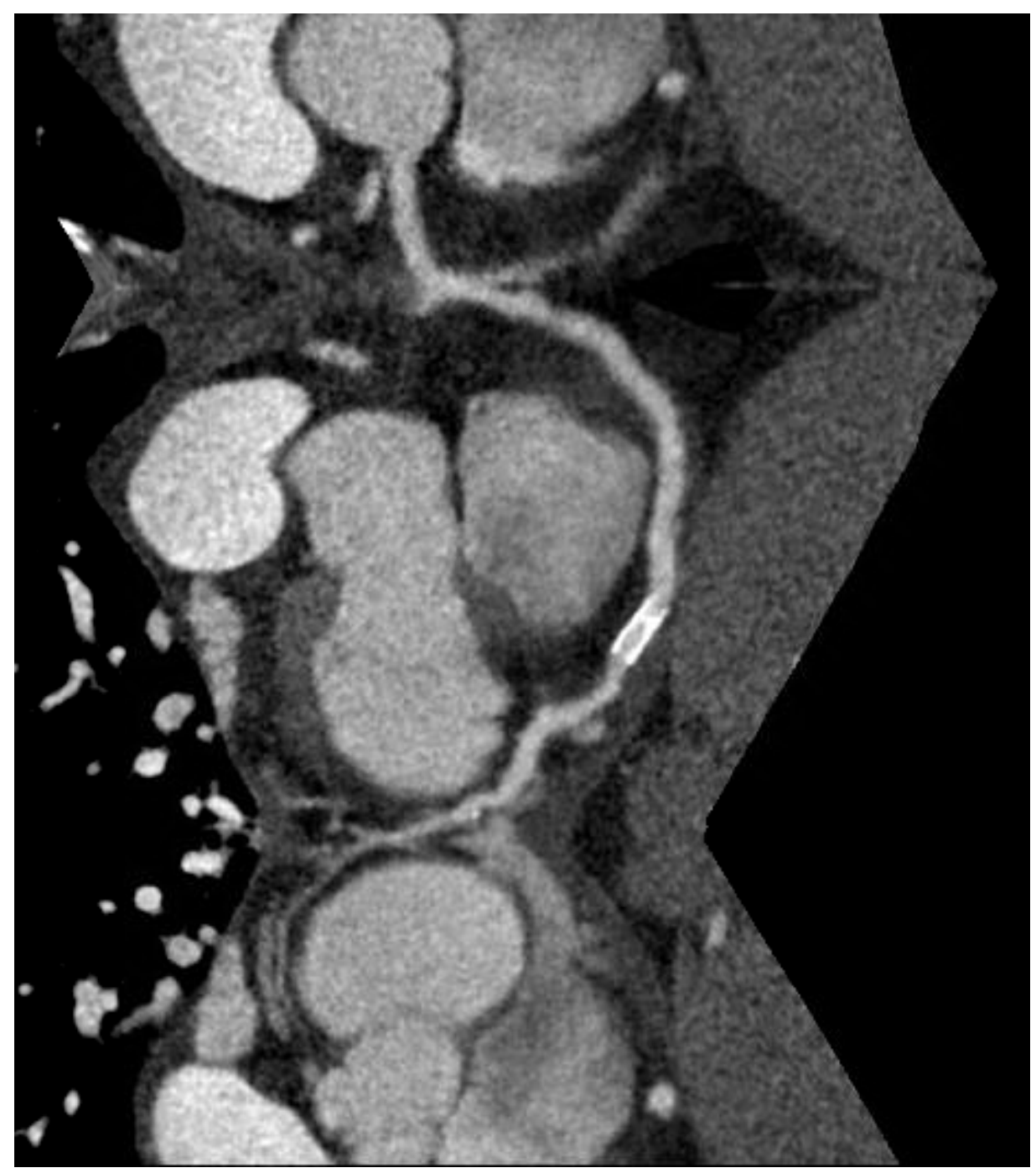

Figure 5. CT coronary angiography demonstrates right coronary artery is superdominant supplying the territory of the obtuse marginals/left circumflex. No LCX

\section{Discussion}

Coronary heart disease is one of the leading causes of death in developed countries (3). Congenital coronary artery anomalies are relatively rare and the literature reported variant prevalence likely biased by the population involved in the studies; reported approximately $0.3 \%-5.6 \%$ [1]. Congenital coronary artery anomalies are associated with the second most common cause of sudden death in young athletes [2]. These anomalies can be further classified into: anomalies that predispose to ischemia, for example, coronary vessels originating from the pulmonary artery, anomalies that do not predispose to ischemia, such as, duplication of LAD, and anomalies that might predispose to ischemia, including congenital absence of LCX [4]. The diagnosis is usually made incidentally during investigation or treatment of heart conditions by CT coronary angiography, interventional coronary angiography, and less commonly by transthoracic or transesophageal echocardiography [4].

In our case, the patient has multiple anomalies. She had an LAD originating from the right sinus of Valsalva (RSV) that is quite rare with a reported incidence of only $0.03 \%$
[5]. The course of the LAD will be one of the following: pre-pulmonic anterior to the right ventricular outflow tract (as in our case) which rarely causes ischemia; retro-aortic posterior to the aortic root, usually benign; inter-arterial between the aorta and pulmonary artery, often associated with grave outcomes; trans-septal subpulmonic course, which rarely causes ischemia; and retro-cardiac in the posterior atrioventricular groove, which predisposes to coronary disease [6]. The other remarkably rare anomaly is absence of the LCX, reported between $0.003 \%$ to $0.067 \%$ [4]. Consequently, the lateral wall will be supplied either by RCA, like in our case, or less commonly from the LAD. It is associated with a potential risk of ischemia likely due to a coronary steal phenomenon [5].

Another interesting aspect about our case is that the patient is young. She has hypertension and the possibility of thrombophilia given the history of membranous glomerulonephritis with proteinuria and mildly elevated homocysteine with positive Dilute Russell's Viper Venom Time. We suspect that the premature acute coronary disease were precipitated by her risk factors and aggravated with her rare coronary anomalies. 


\section{Conclusion}

We report a rare type of congenital CAA; that is an LAD originating from RSV, associated with premature acute coronary syndrome in a young female patient with hypertension and $\mathrm{CKD}$, highlighting the increased risk of myocardial infarction associated with this type of congenital CAA and the technical difficulties during management.

\section{Acknowledgements}

This work is supported, in part, by the efforts of Dr. Moro O. Salifu M.D., M.P.H., M.B.A., M.A.C.P., Professor and Chairman of Medicine through NIH Grant number S21MD012474

\section{References}

[1] Young PM, Gerber TC, Williamson EE, Julsrud PR, Herfkens RJ. Cardiac imaging: Part 2, normal, variant, and anomalous configurations of the coronary vasculature. AJR Am J Roentgenol. 2011; 197: 816-826.

[2] Camarda J, Berger S. Coronary artery abnormalities and sudden cardiac death. Pediatr Cardiol. 2012 Mar; 33(3): 434-8.

[3] Roger VL. Epidemiology of myocardial infarction. Med Clin North Am 2007; 91: 537-52.

[4] Villa AD, Sammut E, Nair A, Rajani R, Bonamini R, Chiribiri A. Coronary artery anomalies overview: the normal and the abnormal. World J Radiol. 2016; 8: 537-555.

[5] Yamanaka O, Hobbs RE. Coronary artery anomalies in 126,595 patients undergoing coronary arteriography. Cathet Cardiovasc Diagn. 1990 Sep; 21(1): 28-40.

[6] Angelini P. Coronary artery anomalies--current clinical issues: definitions, classification, incidence, clinical relevance, and treatment guidelines. Tex Heart Inst J. 2002; 29(4): 271-8.

(C) The Author(s) 2019. This article is an open access article distributed under the terms and conditions of the Creative Commons Attribution (CC BY) license (http://creativecommons.org/licenses/by/4.0/). 